\title{
AUTOMATIC VOLUME CONTROL FOR AIRCRAFT RADIO RECEIVERS
}

\author{
By W. S. Hinman, jr.
}

\section{ABSTRACT}

An automatic volume-control device is described for use primarily in the reception of visual type radio range-beacon signals, the device being easily applied to existing aircraft radio receiving sets. This device operates on the output voltage of the radio receiver, and is provided with a filter unit to prevent operation of the automatic volume control by signals other than those from the range beacon. The controlling voltage is derived from the output of the radio receiver, part of which is rectified and then applied as negative bias to the radio-frequency amplifier. The automatic volume control maintains a substantially constant output voltage for input voltage variations of the order of 5,000 to 1. A distance indicator, operating in conjunction with the automatic volume-control device, is provided to serve as a gage of distance from the transmitting station.

A variation of the automatic volume-control device is given which is intermediate between completely automatic and manual volume control. This semiautomatic volume-control arrangement serves the purpose of simplifying the use of the range beacon on the part of the pilot, and at the same time permits the retention of sense of approach to the beacon station without requiring the addition of a distance indicator.

\section{CONTENTS}

I. Introduction

II. The filter unit.

III. The automatic volume-control device

1. General description

2. Control grid method........

3. Semiautomatic control using control-grid method........

4. Semiautomatic control using screen-grid method

IV. Acknowledgment

\section{INTRODUCTION}

One of the advantages of the visual type radio range beacon ${ }^{1}$ is that automatic volume control may be used in its reception. In the course of a flight on an air route equipped with a radio range beacon, the changes in the input voltage to the receiving set are of the order of 5,000 to 1. This has hitherto necessitated frequent adjustment of receiving set sensitivity by the pilot, so that the output voltage to the indicator might remain fairly constant. These adjustments are increasingly frequent as the airplane approaches the beacon station, since the beacon field intensity then varies most rapidly. The elimination of this need of frequent manual adjustment was the object of this development of automatic volume control.

The development was particularly necessary for the operation of a range-beacon arrangement as part of a system of aids to the blind

1 J. H. Dellinger, H. Diamond, and F. W. Dunmore, Development of the Visual Type Airway Radio Beacon System, B. S. Jour. Research, 4, p. 425; March, 1930. Proc. Inst. Radio Engrs., 18, p. 796; May, 1930. 
landing ${ }^{2}$ of aircraft. In the course of the landing maneuvers, the range of input voltage variations is as great as those encountered during a flight on a radio range beacon. The pilot has no time to adjust volume, since the instruments and the controls of the airplane require his full attention.

The automatic volume control described in this paper was developed to meet a number of practical conditions. One is that it be light in weight. Another is that it be easily applied to existing types of aircraft radio receivers; this points to an automatic volume control operated by the output voltage of the radio receiver, for any other means for automatically controlling the voltage would necessitate difficult alterations in the radio receiver. Another condition is that when automatic volume control is used on the visual radio

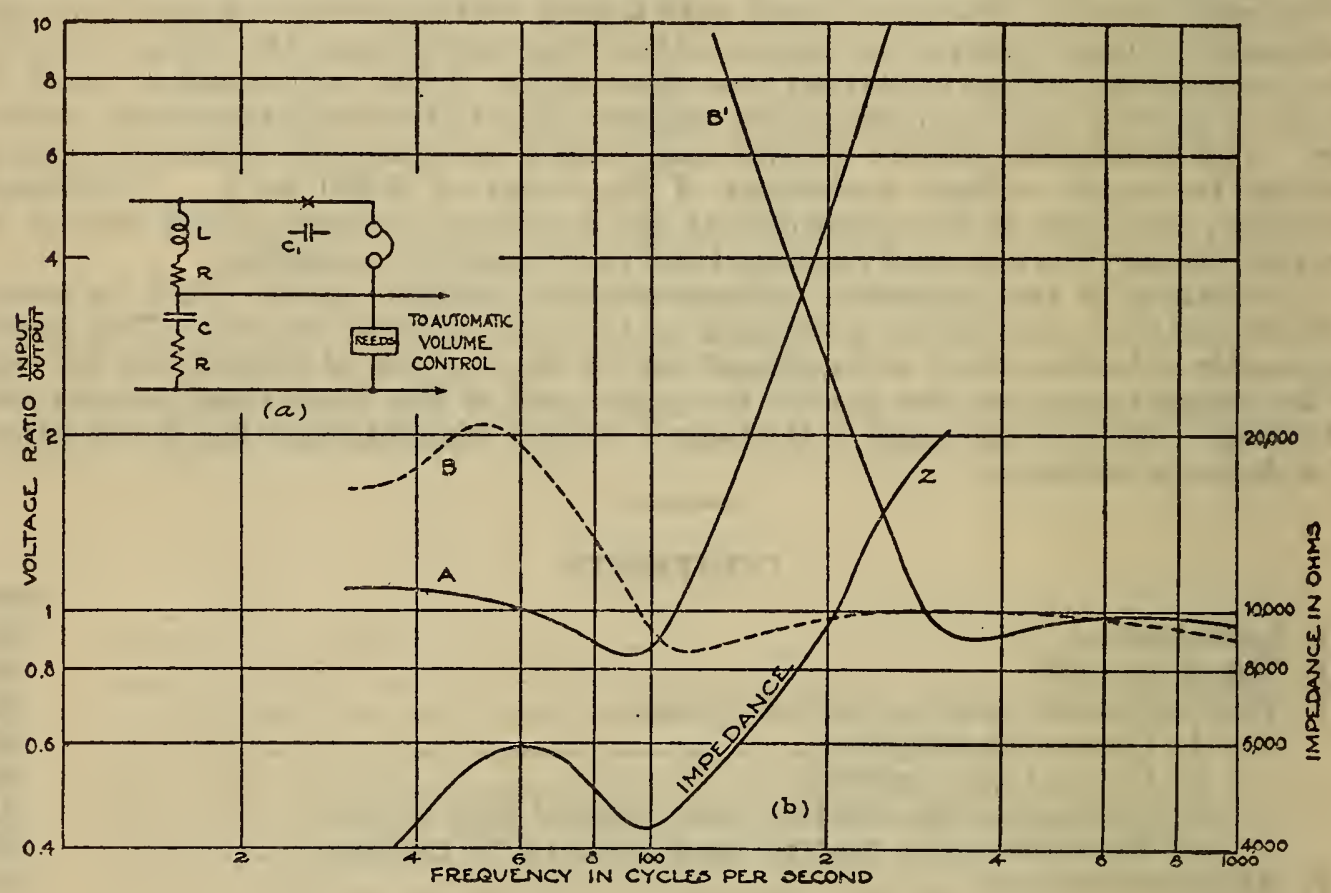

Figure 1.-Performance graphs of filter unit

range beacon, it must be operated by the signal of the beacon station only, in order that the ability of the course indicator (which may be either a reed indicator or a reed converter ${ }^{3}$ ) to distinguish between that signal and any interfering signal be preserved. Here, again, an automatic volume control operating on the output of the radio receiver offers a simple means of attaining this end. A filter unit may be used in the output of the radio receiver so that the signals of the radio range beacon only are applied to the automatic volume control.

\section{THE FILTER UNIT}

Since the frequencies of the visual radio range beacon are between 65 and 108 cycles, a low pass filter unit may be used to separate these signals from any other signals. However, the radio receiver must

\footnotetext{
2 H. Diamond and F. W. Dunmore, A Radiobeacon and Receiving System for Blind Landing of AirCraft, B. S. Jour. Research, 5 , pp. 897-931; October, 1930

F. W. Dunmore, A Course Indicator of Pointer Type for the Visual Radio Range-Beacon System, B S, Jour. Research, 7, p. 147.
} 
also be used for speech reception, and sometimes for simultaneous reception of both speech and radio range-beacon transmissions. ${ }^{4}$

In order that speech may be received, a high pass filter unit also is necessary. The use of two such filter units makes weight and size important in the filter unit design.

For this reason the filter circuit arrangement shown in Figure 1 (a) was developed. Condenser $C$ forms a parallel resonant circuit with the reed indicator, and is adjusted to resonate at about 60 cycles. Inductor $L$ forms a series resonant circuit with the parallel circuit formed by condenser $C$ and the reed indicator, and is adjusted

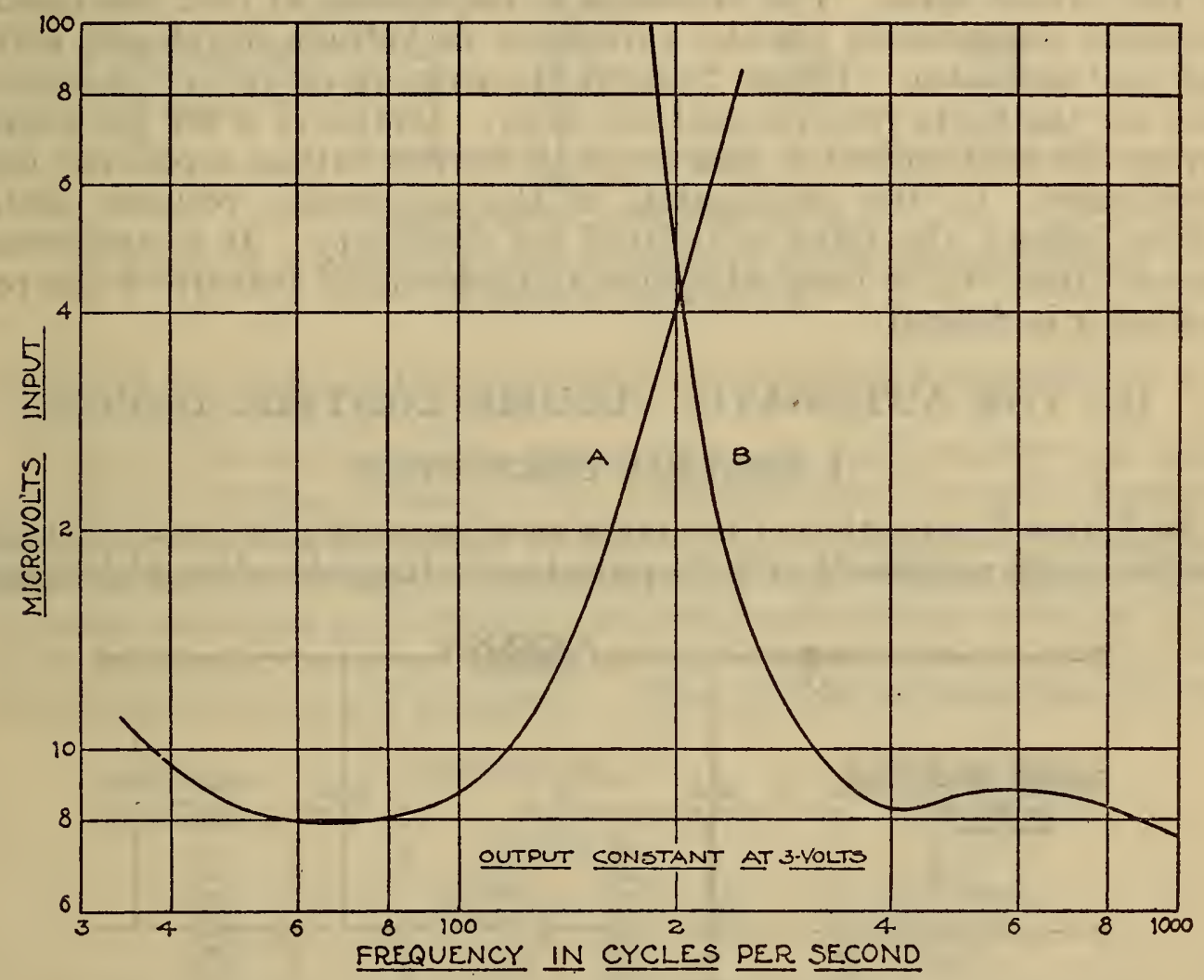

FIGURE 2.-Over-all performance graph of radio receiver and filter unit

to resonate at about 90 cycles. These two resonance points are shown in graph $Z$ of Figure $1(b)$, which shows the input impedance of the filter. The impedance of inductor $L$ is low at frequencies lower than the point of series resonance, but is increasingly high at higher frequencies. The impedance of the parallel resonance circuit is, of course, high at frequencies near its point of resonance, but increasingly low at higher frequencies. The lower frequencies develop highest voltage across condenser $C$ and the reed indicator, while the higher frequencies develop highest voltage across inductor $L$. The head telephones are connected across inductor $L$, but have little effect on the filter characteristics because of their high impedance. Resistors $R$ reduce the resonance effects of the filter so that the voltage developed across the reed indicator is uniform over the required frequency range. The graphs of Figure $1(b)$ show the ratio of the voltage applied to the filter, to the voltage applied to the reed

F. G. Kear and G. H. Wintermute, A Simultaneous Radiophone and Visual Range Beacon for the Airways. Will appear in B. S. Jour. Research for August. 
indicator and the head telephones at various frequencies. Referring to graph $A$, it will be noted that practically no voltage of frequencies higher than 250 cycles is developed across the reed indicator. However, low frequency cut-off for the head telephones (see graph $B$ ) is very poorly defined. Small condenser $C_{1}$ is placed in series with the head telephones at $X$ of Figure $1(a)$. This sharpens the lowfrequency cut-off for the head telephones as shown by graph $B^{\prime}$. Since speech depends largely on the higher frequencies for intelligibility, the performance indicated in $B^{\prime}$ is satisfactory. Graph $Z$ of Figure $1(b)$ shows the impedance presented by the filter to the plate of the output tube. The variation in impedance at reed frequencies tends to compensate for the variations in voltage developed across the reed indicator. Figure 2 shows the over-all frequency characteristic for the radio receiver and the filter. Graph $A$ is for the output across the reed indicator, and graph $B$ for the output across the head telephones. In the description of the automatic volume control which follows, the filter is omitted for simplicity. It is understood that a filter will be used whenever the selectivity feature of the reed indicator is desired.

\section{THE AUTOMATIC VOLUME CONTROL DEVICE}

\section{GENERAL DESCRIPTION}

In Figure 3, a portion of the radio receiver output is passed through copper-oxide rectifier $Q$, and the pulsating; voltage developed is changed

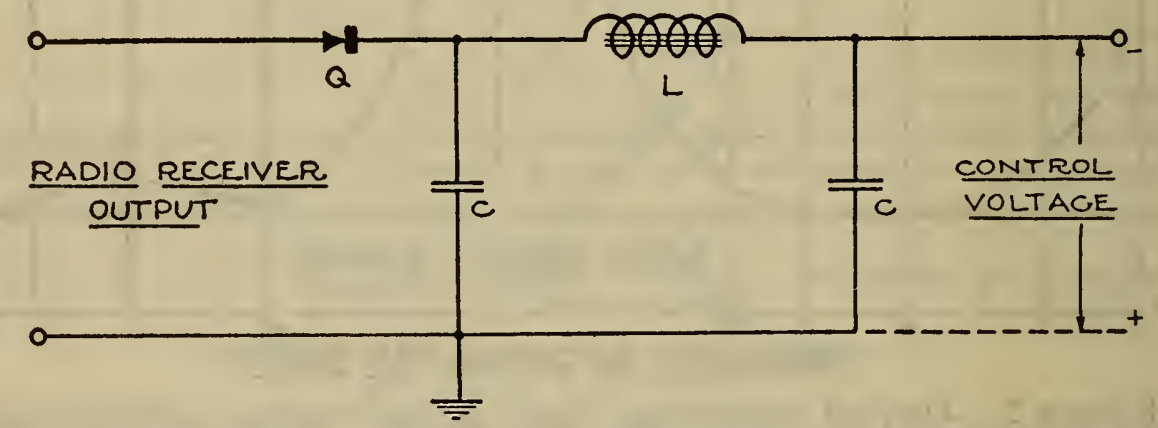

FIGURE 3.-Electrical circuit arrangement of automatic control unit

to nearly steady direct voltage by condensers $C$ and inductor $L$. This voltage is applied to the radio-frequency amplifier in such a way that it decreases the sensitivity of the radio receiver. Since this control voltage is directly proportional to the receiving set output voltage, any increase in input voltage to the radio receiver automatically increases the controlling voltage, thereby tending to oppose the normal increase in output voltage. Thus the output voltage of the radio receiver is held substantially constant for varying in put voltages.

Three methods have been developed for applying automatic volume control to aircraft radio receivers. The advantages of each are shown.

\section{CONTROL-GRID METHOD}

In Figure 4, the output voltage of the radio receiver is applied to a voltage divider $P$ which applies part, or all, of this output voltage to rectifier $Q$. The output of $Q$ is filtered and applied as a direct voltage of negative polarity to the control grids of the radio-frequency am- 
plifier. Voltage divider $P$ is used for controlling the output voltage. Its setting determines the degree of automatic control to be used. Rectifier $Q$ is a high resistance, half-wave, copper-oxide rectifier, with low-current capacity. In the filter, resistor $r$ is used in place of the usual inductor to save weight and space. Since the control grids of the radio-frequency tubes in the receiving set draw no power from the rectifier, resistance is as effective as inductance. Switch $S$ is

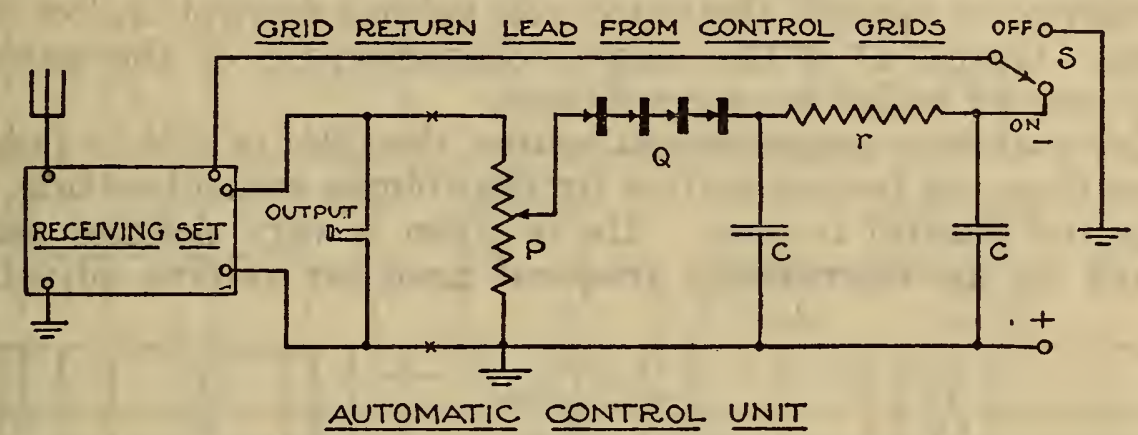

FIGURE 4.-Electrical circuit arrangement of automatic control unit applied to radio receiver

provided to allow the receiving set to be operated either with or without automatic volume control.

Figure 5 shows the means employed in the radio receiver for applying the controlling voltage to the control grids of the radio-frequency amplifier. In most aircraft receiving sets, the means for supplying this controlling voltage is already part of the receiver design, though in some cases the grid resistor and condenser shown must be added. The automatic volume control should operate on as many radiofrequency tubes as are available. The control characteristic becomes

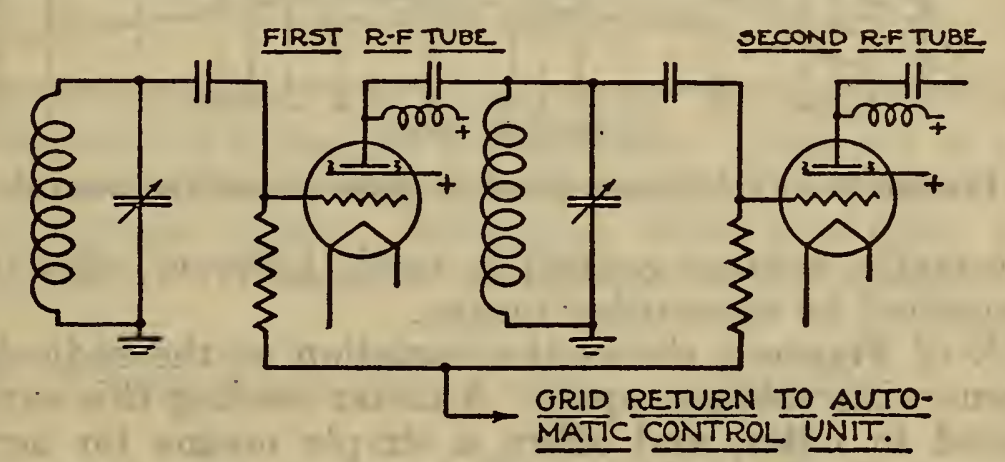

FIGURE 5.-Method of applying control voltage to radiofrequency control grids

flatter as more controlling tubes are used. It is necessary that care be taken in the design of the filter for the automatic volume control. At high input voltages a flutter is apt to be produced in the output of the radio receiver. This flutter is a reaction phenomenon produced by remodulation of the radio-frequency carrier by the small a. c. component of the controlling voltage.

Figure 6 gives graphs showing the characteristics of the automatic volume control when applied to a radio receiver consisting of two stages of radio-frequency, a detector, and two stages of audiofrequency. $A$ is the normal overload graph without automatic volume control. $B$ is a graph showing output voltage for varying input volt- 
ages when automatic volume control is used and corresponds to maximum setting of voltage divider $P$ of Figure 4. $C$ is a graph taken with voltage divider $P$ set at less than its maximum position so that the output voltage of the receiver is greater than the controlling voltage of the automatic volume control. Where it is desired that the receiving set be controlled at lower output voltages, a step-up transformer must be inserted at $X X$ (fig. 4 ) so that sufficient controlling voltage may be developed to operate the automatic volume control on low output voltages. Graph $D$ is the output characteristic of the automatic volume control under these conditions.

In flying along a range-beacon course, the pilot is able to judge the distance from the beacon station by the volume control setting, when full manual control is used. $\mathrm{He}$ is given a very definite sense of approach by the increasingly frequent need for volume adjustment.

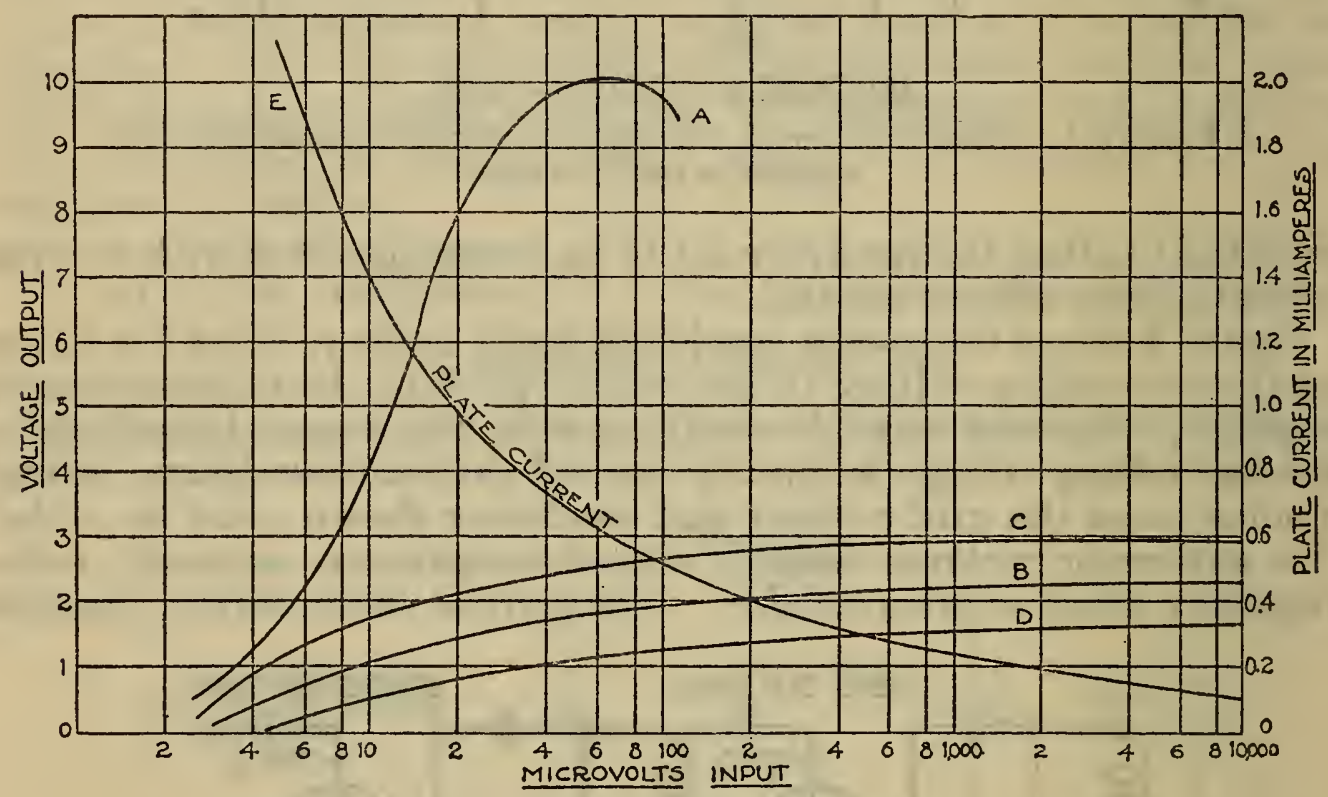

FraURe 6.-Performance graph of automatic volume control

When automatic volume control is used, however, this indication must be supplied by some other means.

Graph $E$ of Figure 6 shows the variation of the radio-frequency plate current with voltage input. A meter reading this current may be calibrated in miles, and offers a simple means for accomplishing distance indication. However, the greatest need for a distance indicator occurs at high input voltages, when the pilot is nearing the beacon station. The current is then low, and variations of input voltage cause very little change in current. This makes accurate reading of the meter difficult. To remedy this defect, a lower current meter provided with a relay-operated shunt to protect the meter at high current values may be used. This shunt may be calibrated, if desired, to read higher values of current. The meter may also be used as a tuning meter, though its use for this object is optional, the amplitude of reed vibration normally serving this purpose.

The greatest value of the automatic volume control is its use when the received signals are of high field in tensity; that is, when the pilot is close to the beacon station. It is then that the adjustments of volume become so frequent that they are very troublesome, if not 
impossible. The automatic volume control may be used, however, at all times save when the full sensitivity of the radio receiver is required. The sensitivity of the radio receiver is reduced slightly by the automatic control unit. This has been shown by the graphs of Figure 6. Since either automatic or menual control of volume may

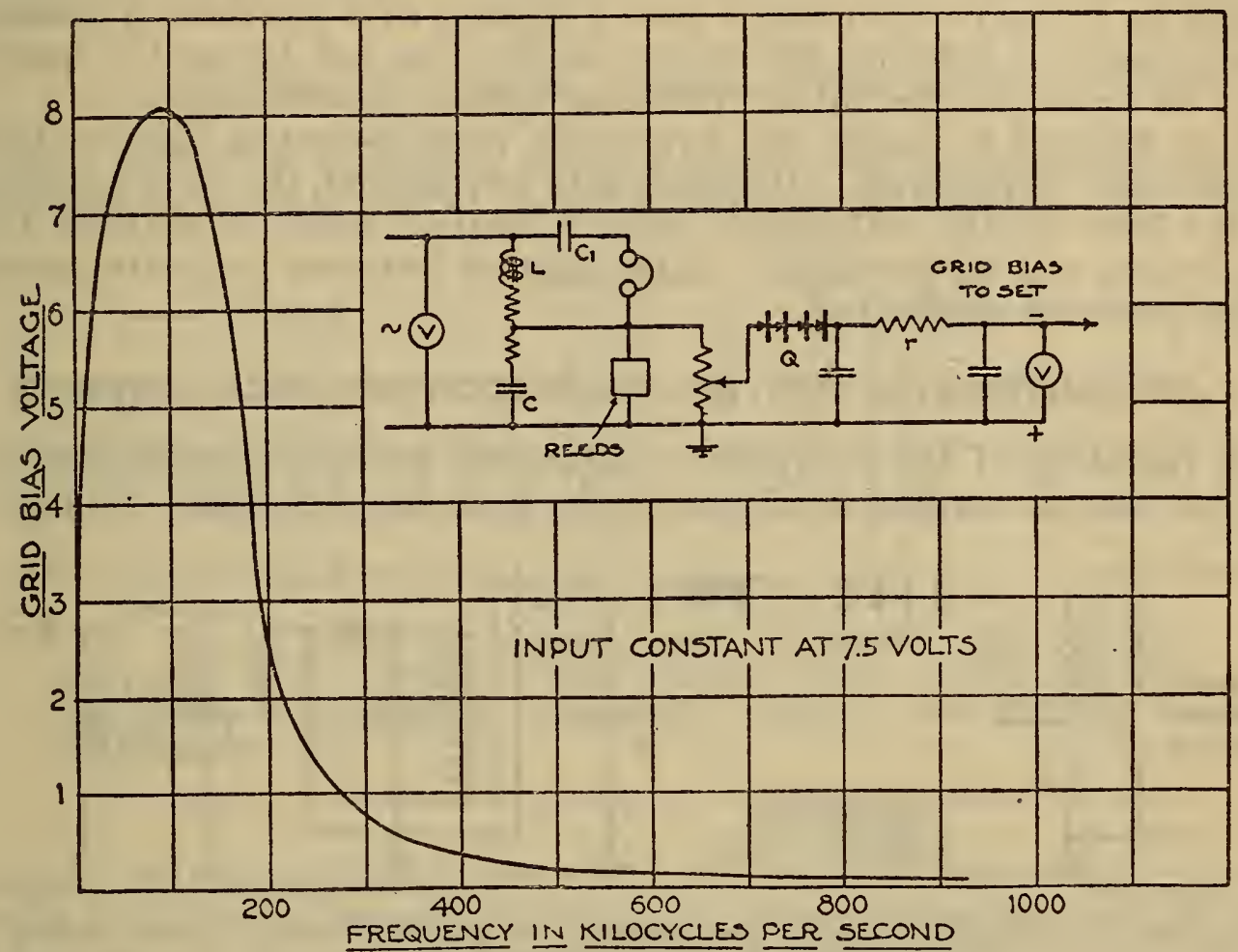

FIGURE 7.-Variation of grid bias voltage with frequency for filter and automatic control units

be used at will (by means of switch $S$, fig. 4) the pilot may use the type of control best suited to the occasion. Figure 7 is a graph in which the controlling voltage developed by the automatic volume control is plotted against frequency, when the filter previously described is used. The circuit arrangement is also shown in Figure 7.

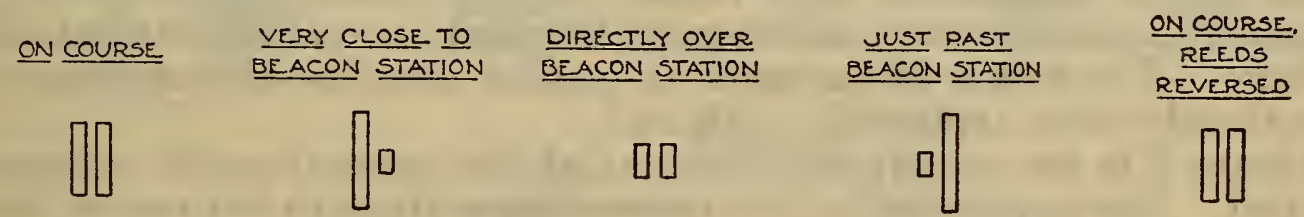

FIGURE 8.-Localizing action of reed indicator over range-beacon tower

The freedom from interfering signals obtained through the use of the filter circuit is apparent.

The usual zero-signal zone directly over the beacon station is modified when automatic volume control is used. When the pilot is nearly over the beacon station, one reed decreases in amplitude until it is at rest. As he passes over the loop antennas of the radio range beacon, the reeds reverse; that is, the reed formerly at rest rises to the amplitude of the vibrating reed, while the reed formerly vibrating decreases in amplitude until it is at rest. Then, as the pilot proceeds on the back course, the reeds become equal again. Figure 8 shows the 
positions of the reeds, in the order named, as the pilot passes over the beacon station.

It is worthy of note that the flutter phenomenon previously described may be used to advantage in flying the visual radio range. If this flutter be introduced, and made to occur at the field intensity of the visual range beacon at 4 miles, the pilot is warned that he must watch for the indication which occurs directly over the beacon station. This means for locating the beacon station can not be used if speech is to be received, the flutter rendering speech unintelligible.

It is difficult to design the automatic volume-control filter so that speech may be received. If speech is to be received, the time constant of the filter of the automatic volume control must be reduced to a minimum, and all possibility of interaction between radio-frequency tubes must be eliminated.

\section{SEMIAUTOMATIC CONTROL USING CONTROL-GRID METHOD}

A variation of the completely automatic volume control just described was developed to eliminate the need for a distance indicator.

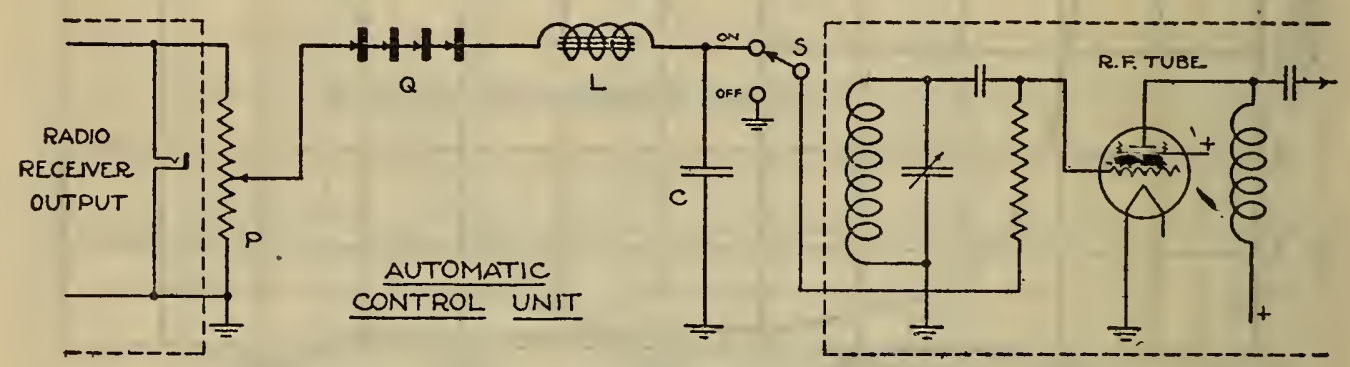

FIGURE 9.-Electrical circuit arrangement for semiautomatic volume control operating on the control grids

It is a semiautomatic volume control which does not change the operation of the radio receiver in any way, but supplements the manual volume control, reducing to approximately one-fifth the number of adjustments necessary over a given range of input voltage. It gives a very definite sense of approach to the beacon station without the use of the special distance indicator previously mentioned, since the reeds rise in amplitude more rapidly as the beacon station is approached, and necessitate increasingly frequent manual adjustments of volume. The action of the reeds in passing over the beacon station has already been explained. (Fig. 8.)

Figure 9 is the circuit arrangement of the semiautomatic volume control. The application of the controlling voltage to but one of the radio-frequency tubes eliminates any possible interaction between tubes. The filter of the automatic control unit consists of inductor $L$ and condenser $C$, and has a low time constant (that is, time required for developing the control voltage). In all other respects the semiautomatic volume control is similar to the completely automatic volume control previously described. Since voltage divider $P$ regulates the value of the controlling voltages, the extent to which the automatic control unit controls the volume may be varied at will. Figure 10 is a graph which gives the characteristics of the semiautomatic volume control when voltage divider $P$ applies about one-third of the output voltage to the control unit. It is also a comparison of semi- 
automatic and manual volume control in number of adjustments necessary to maintain a constant voltage output.

Any signal may be received with this semiautomatic volume control. The radio receiver sensitivity is not impaired. The radio receiver may be used either with or without semiautomatic volume control.

\section{SEMIAUTOMATIC CONTROL USING SCREEN-GRID METHOD}

This method is somewhat simpler, with regard to changes necessary in the radio receiver proper, than the control grid method. When the

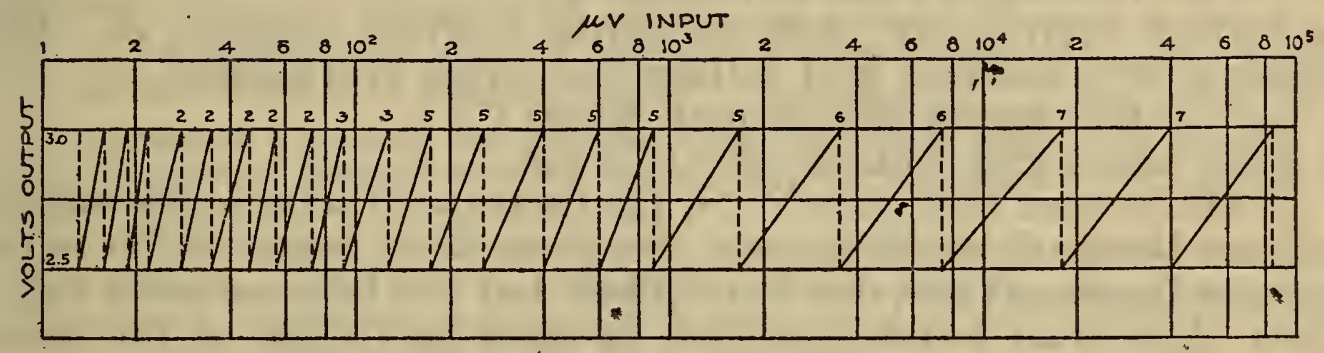

DOTTEO LINES SHOW ADJUSTMENTS NECESSARY FOR KEEPING

OUTPUT AT LEVEL SHOWN
LINE SHOW ADJUSTMENTS NECESSARY WHERE FULL MANUAL
CONTROL IS USED.

FIGURE 10.-Performance graphs for semiautomalic volume control

manual volume control is one which varies the voltage on the screen grids of the radio-frequency tubes, the screen grid power supply lead is broken and the voltage developed by the automatic volume control is placed in series, but opposing the screen grid voltage. Figure 11 shows the circuit arrangement. Since the screen grids require current, care must be taken that the automatic volume control does not absor'b too much power from the radio receiver output. A step-up

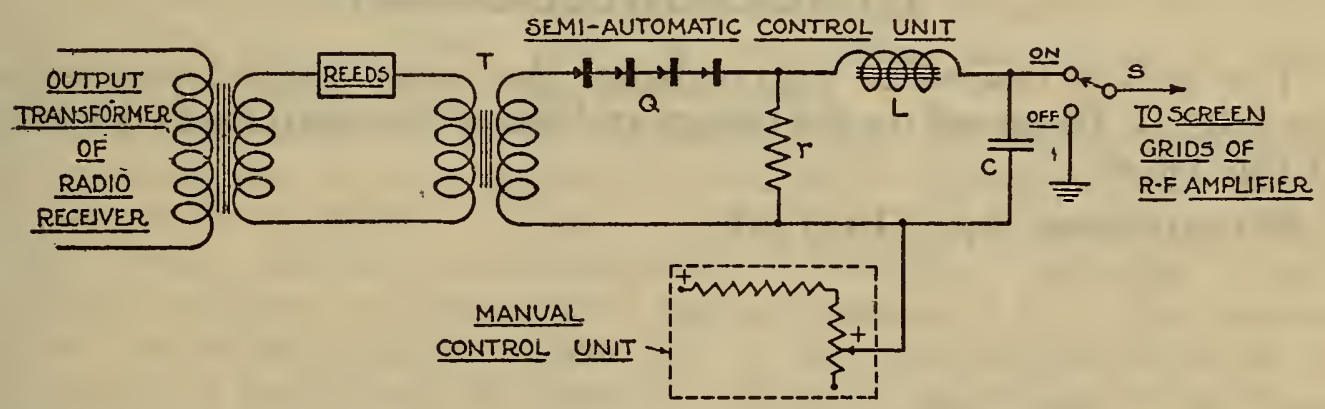

FIGURE 11.-Electrical circuit arrangement for semiautomatic volume control operating on screen grids

transformer $T$ is placed in series with the output load. Since this transformer has low impedance and since it raises the output load impedence, the power loss due to the automatic volume control is small.

In Figure 11, the output of the rectifier $Q$ is shunted by a resistor $r$. This resistor supplies a path for the screen grid current, and makes the load on the secondary of transformer $T$ pure resistance. This is reflected back to the low impedance primary as a pure resistance load of low impedance. The voltage divider, used to determine the con- 
trolling voltage of the automatic volume control is not used. The controlling voltage is determined by the constants of transformer $T$. The operation of this automatic volume control is similar to that previously described. In this method for automatically controlling the volume, there is an inherent characteristic which makes supplementary adjustment of volume necessary. It is best explained by reference to Figure 11.

Let $E=$ voltage of movable arm of manual control unit.

$i=$ screen-grid current when no current is produced by rectifier.

Screen grid voltage $=E-i r$.

$\dot{i}_{1}=$ current in $r$ due to rectifier $Q$.

A voltage drop $i_{1} r$ is produced across $r$ which opposes $E$. This causes a drop in screen grid voltage and screen grid current.

Let $i^{\prime}=$ new screen grid current, where $i^{\prime}<i$.

Then screen grid voltage $=E-\left(i^{\prime}+i_{1}\right) r$.

It will be seen that since $i^{\prime}<i$, the decrease in screen-grid voltage will not be equal to the increase of voltage drop across $r$ (due to the increase in current in $r$, due to rectifier), but will be some lesser value. Thus, there is an action that tends to raise the voltage on the screen grids as the current produced by the rectifier tends to lower this voltage, and there is a gradual rise of voltage output of the receiving set with an increase of input voltage. If resistor $r$ could be made to vary with the current through it, so that its resistance is high at low-current values, and low at high-current values, it would tend to compensate for this tendency toward a rise of screen-grid voltage. A copper-oxide rectifier used as a resistor fulfills this requirement.

The volume control characteristics of this semiautomatic volume control are the same as the characteristics given in Figure 10 for the semiautomatic volume control using the control grid method. It may be used on any signal, and has little effect on the sensitivity of the radio receiver.

\section{ACKNOWLEDGMENT}

The author desires to acknowledge the many helpful suggestions made by $\mathrm{H}$. Diamond on the design and use of the equipment described in this paper.

Washington, April 18, 1931. 